\title{
International Journal of Managing Information Technology (IJMIT)
}

ISSN: 0975- 5586 [Online]; 0975-5926 [Print]

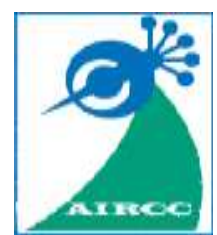

ISSN: $0975-5586$ (Onfine)

$0975-5926$ (Print)

\section{IJMIT}

International Journal of Managing

Information Technology

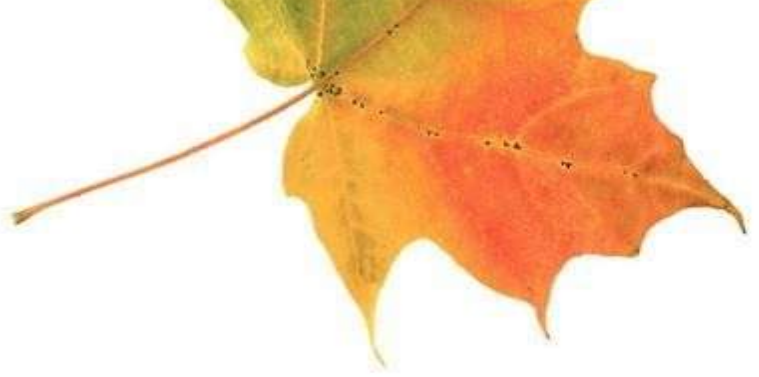

https://airccse.org/journal/ijmit/ijmit.html 


\section{SCOPE OF THE JOURNAL}

The International Journal of Managing Information Technology (IJMIT) is a quarterly open access peer-reviewed journal that publishes articles that contribute new results in all areas of the strategic application of information technology (IT) in organizations. The journal focuses on innovative ideas and best practices in using IT to advance organizations - for-profit, non-profit, and governmental. The goal of this journal is to bring together researchers and practitioners from academia, government and industry to focus on understanding both how to use IT to support the strategy and goals of the organization and to employ IT in new ways to foster greater collaboration, communication and information sharing both within the organization and with its stakeholders. The International Journal of Managing Information Technology seeks to establish new collaborations, new best practices, and new theories in these areas

\section{Topics of interest within the scope of this issue include, but are not limited to, the following} areas:

Authors are solicited to contribute to the journal by submitting articles that illustrate research results, projects, surveying works and industrial experiences that describe significant advances in the areas of information technology and management.

- Education and Information Technology

- Electronic Billing Systems

- Electronic Voting \& E-Government Systems

- Environmental Management Technologies

- Financial Market \& Trading Technologies

- Financial Risk Management Systems

- Green Technologies

- Health Care Information Systems and Technologies

- High-Definition TV Systems \& Standards

- Information Goods \& Digital Products

- Information Privacy \& Digital Security

- Information Technology for Economic \& Social Development

- Location-Based Systems \& Services

- Management Fashion Developments

- Market Operations \& Exchange Solutions
- Mobile Telecommunication Systems

- Nomadic Computing Service Innovations

- Online Auctions and Market Mechanisms

- Online Instruction \& Digital Learning Aids

- Open Software and Systems Innovations

- $\quad$ Service-Oriented Systems \& Technologies

- Statistical Methods for Information Technology Adoption

- Technology Ecosystem Forecasting Tools

- Technological Innovation-Led Performance

- Technology Stack, Infrastructure Changes

- Vendor-Managed Inventory, Supply Chain

- Vertical Information Systems Standards

- Web 2.0 Technologies

- Yield Management and Revenue Control 


\section{Editorial Board}

\section{Editor In Chief:}

C.E. Laborde Professor of Management

David C. Wyld, Southeastern Louisiana University,USA

\section{Editor In Chief:}

David C. Wyld, Southeastern Louisiana University,USA

\section{Associate Editors}

Chihiro Watanabe, University of Jyvaskyla, Finland

Sitalakshmi Venkatraman, Melbourne Polytechnic, Australia

\section{Editorial Board}

- Adnane Cabani, Embedded Electronic Systems Research Institute, France

- Adolfo Alberto Vanti, Universidade do Vale do Rio dos Sinos, UNISINOS, Brazil

- Ahmed M. Khedr, Zagazig University, Egypt

- Ahmed Y. Nada, Al - Quds University, Palestine

- Akbar Hussain D.M, Aalborg University, Denmark

- Alejandro Regalado Mendez, Universidad del Mar, Mexico

- Ali AlSoufi, University of Bahrain, Bahrain

- Amala VijayaSelvi Rajan, Higher Colleges of Technology - Dubai Women's College, UAE

- Andree Carrier Taylor, Southeastern Louisiana University,USA

- Anjali Thukral, University of Delhi, India

- Anna Nicholson Bass, Southeastern Louisiana University,USA

- Antonio Rodriguez-Martinez,Universidad Autonoma Del Estado de Morelos, Mexico

- Aristides R. Baraya, Southeastern Louisiana University,USA

- Atif Farid Mohammad, University of North Dakota, USA

- Avinash Waikar, Southeastern Louisiana University,USA

- Aymé Perdomo Alonso, University of Informatics Sciences, Cuba

- Binod Kumar Pattanayak, Siksha O Anusandhan University, India 
- Bouakkaz Mustapha, University Amar Telidji Laghouat, Algeria

- Buket D. Barkana, University of Bridgeport, USA

- Carlos Andre Reis Pinheiro, Dublin City University, Ireland

- Chris Panagiotakopoulos, University of Patras, Greece

- Chunxiao Li, Waseda University, Japan

- Connie Budden, Southeastern Louisiana University,USA

- Danaysa Macias Hernandez,Universidad de Ciencias Informaticas, Cuba

- Daniela Lopez De Luise, Universidad de Palermo, Argentina

- David B. Bracewell, General Electric Global Research, USA

- Denivaldo Lopes Federal, University of Maranhao, Brazil

- DimitriosTomtsis, Technical and Educational Institute of West Macedonia,Greece

- Dinh Tran Ngoc Huy, Banking University HCM city Viet nam

- Dong-Seong Kim,Duke University, USA

- Elaheh Pourabbas, National Research Council (CNR), Italy

- Elboukhari Mohamed,University Mohamed First,Morocco

- Elmir Babovic,Univeristy of Mostar, Bosnia and Herzegovina

- Fawzi Albalooshi, University of Bahrain-Sakhir Campus,Bahrain

- Gajendra Sharma, Kathmandu University, Nepal

- Gerardo S. Doroja, Xavier University - Ateneo de Cagayan, Philippines

- Guoliang Zeng, Arizona State University, USA

- Hariharan S, Shadan Women's̄ College of Engineering and Technology, India

- Hariharan S, Pavendar Bharathidasan College of Engineering and Technology, India

- Harilton da Silva Araujō, State University of Piauí- UESPI, Brazil

- Harrison Hao Yang, State University of New York at Oswego, USA

- Hassan Jorfi,Universiti Teknologi Malaysia (UTM), Malaysia

- Hector Gonzalo Barbosa Leon,Universidad de Salamanca, Espana

- Ian McAndrew, Capitol Technology University, USA

- Inci Saricicek, Eskisehir Osmangazi University, Turkey

- Indrajit Mandal, Faculty Member Visvesvariah Institute of Technology - Bangalore, India

- Issac Biju, Teesside University, UK

- Jain Y.K, Samrat Ashok Technological Institute, India

- Jan Genci, Technical University of Kosice, Slovakia

- Jaroslav Abaffy, Slovak University of Technology, Bratislava

- Jayant Mishra ,Rajiv Gandhi Proudyogiki Vishwavidyalaya, India 
- Jorge Bernardino, Instituto Superior de Engenharia de Coimbra, Portugal

- Jose Francisco Briones de la Torre, Universidad Politecnica de Aguascalientes, Mexico

- Julie Nunenmacher, Southeastern Louisiana University,USA

- Jyotirmay Gadewadikar, Alcorn State University, USA

- Kapil Chaturvedi, Rajiv Gandhi Technical University, India

- Katarina Jelemenska, Slovak University of Technology, Slovak Republic

- Kun Ren, Yale University, USA

- Labeeb, Misr Academy for engineering and technology (MET), EGYPT

- Lotfollah Najjar, University of Nebraska, Omaha

- Lylia Abrouk, University of Burgundy, France

- Madhuri Rao, Mumbai University, India

- Maniza Hijab, Osmania University, India

- Maria Emmalyn Asuncion De Vigal Capuno, Future University, Sudan

- Mario Ciampi, Institute for-High Performance Computing and Networking, ICAR, Italy

- Marius Marcu, Politehnica University of Timisoara, Romania

- Masaki Murakami, Okayama University, Japan

- Massimo Esposito, Institute for High Performance Computing and Networking (ICAR) of the National Research Council of Italy (CNR), Italy

- Mazeyanti M. Ariffin, Universiti Teknologi Petronas, Malaysia

- Michael C. Budden, S̄outheastern Louisiana University,USA

- Michael Jones, Southeastern Louisiana University,USA

- Michel Owayjan, American University of Science \& Technology, Lebanon

- Ming-Chang Lee, National Kaohusing University of Applied Sciences, Taiwan

- Minh Huynh, Southeastern Louisiana University,USA

- Mohamed abd el Fattah Mohamed, Helwan University ,Egypt

- Mohamed Firdhous, University of Moratuwa, Sri Lanka

- Mohammad Hadi Zahedi, Ferdowsi University of Mashhad, Iran

- Mohammed A. Otair, Amman Arab University, Jordan

- Monika Verma, Punjab Technical University,India

- Nadezhda N. Pokrovskaia, Herzen State Pedagogic University, Russia

- Nawapon Kewsuwun, Prince of Songkla University, Thailand

- Nguyen Van Sinh, Vietnam Academy of Science and Technology, Vietnam

- Nirbhay Chaubey, Institute of Science \& Technology for Advanced Studies \& Reasearch (ISTAR), India

- Pacha Malyadri, Center for Economic and Social Studies (CESS), India 
- Nour Eldin Elmadany, Arab Academy for Science and Technology, Egypt

- Ognjen Kuljaca, Alcorn State University,USA

- Oleg Shcherbina, University of Vienna, Austria

- Oluwatope Ayodeji Oludola, Obafemi Awolowo University, Nigeria

- Othon Marcelo Nunes Batista, Universidade Salvador, El Salvador

- Paola Monachesi, Utrecht University, The Netherlands

- Paul Sagayda, Donbass State Engineering Academy, Ukraine

- Rafiqul Zaman Khan̄, Aligarh Muslim University, India

- Rahmath Safeena Abdullah, Taif University, Saudi Arabia

- Rahul Chauhan, Parul University, India

- Rakesh Kumar Mishra, Feroze Gandhi Institute of Engineering and Technology, India

- Ramayah Thurasamy, Universiti Sains Malaysia, Malaysia

- Rasha Abd Elaziz, Arab Academy for Science and Technology and Maritime Transport, Egypt

- Reza Ebrahimi Atani, University of Guilan, Iran

- Richard David Ramsey, Southeastern Louisiana University,USA

- Richard Millham, University of Bahamas, The Bahamas

- Rick Leopoldi, RL Information Consulting, LLC, USA

- Sachin Subhash Patil; Shivaji University, India

- Saeid Jorfi, Universiti Teknologi Malaysia(UTM) Malaysia

- Sang H. Lee, Southeastern Louīsiana University,USA

- Sat Sharma, University of Manitoba, Canada

- Satria Mandala, Maliki Stäte Islamic University, Indonesia

- Sherif Rashad, Moreheād State University, USA

- Shubhamoy Dey, Indian Institutes of Management, India

- Srinivas Acharyulu P.V, National Thermal Power Corporation, India

- Teri L. Root, Southeastern Louisiana University,USA

- Thair Khdour, Albalqa Applied University, Jordan

- Thomas Yang, Embry-Riddle Aeronautical University, USA

- Tien D. Nguyen, Coventry University United Kingdom, UK

- Titus Bitek Watmon, University of East London, United Kingdom

- Vipin Tyagi, Jaypee University of Engineering and Technology, India

- Wahiba Ben Abdessalem Karaa, of Tunis Institut Superieur de Gestion Cite, Tunisia

- Walid Qassim Qwaider, Majmaah University, Saudi Arabia 
- William E. Westermann, Southern Methodist University, USA

- Xin Zhou, Kyushu University, Japan

- Yan Wang, Kennesaw State University,USA

- Yogendra Kumar Jain, Samrat Ashok Technological Institute, India

- Yuval Cohen, The Open University of Israel, Israel

- Zeenath Reza Khan, University of Wollongong, Dubai, UAE

- Zheng (Jeremy) Li, University of Bridgeport, USA 


\section{Paper Submission \& Manuscript preparation Guide}

Authors are invited to submit papers for this journal through Email: ijmitjournal@aircconline.com or through Submission System. Submissions must be original and should not have been published previously or be under consideration for publication while being evaluated for this Journal. For paper format download the templatein this page

\section{Manuscript Template}

\section{Review Process}

Submissions are accepted for review with the understanding that the same work has been neither submitted to, nor published in, another publication. Simultaneous submission to other publications will result in immediate rejection of the paper. Papers are not within the journal scope will be rejected immediately after the pre review process. All manuscripts will be subject to a well established, fair, unbiased peer review and refereeing procedure, and are considered on the basis of their significance, novelty and usefulness to the Journals readership. The reviewing structure will always ensure the anonymity of the referees $\&$ it will be reviewed by 3 experts in the field. The review output will be one of the following decisions:

1. Accept

2. Accept with minor changes

3. Weak Accept with major changes

\section{Reject}

The review process may take approximately two three months to be completed. The Editor reserves the right to reject a paper if it does not meet the aims and scope of the journal, it is not revised well.

\section{Copy Right Form}

After submitting final manuscript, you can get copy right form from AIRCC secretary

\section{Special Issue Proposal}

IJMIT invite proposals for special issues on topics that fall within the scope of this journal.

Please email your proposal to secretary@airccse.org.

\section{Contact Us}

Here's where you can reach us : ijmitjournal@aircconline.com or ijmit@airccse.org 


\section{INDEXING}

\section{Abstracting \& Indexing Services:}

The articles of IJMIT are indexed/abstracted in the following index services.

$\underline{\text { The Elektronische Zeitschriftenbibliothek EZB }}$

$\underline{\text { Genamics JournalSeek }}$

$\underline{\text { World Journal Clout Index (WJCI) }}$

ProQuest

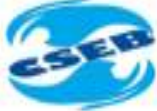

pubget

PKP

PUBLIC

KNOWLEDGE

PROJECT

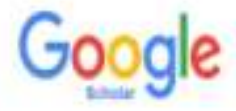

Scribd.

docstac Citeseerk

5. WorldCat

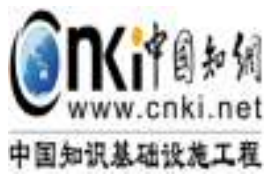

SCIIUS

brescietifintinationatly

EBSCO

PUAL15HIN 
International Journal of Managing Information Technology (IJMIT)

\section{Google Scholar Indexing}

H -Index - 24, Citations 2262, i10-Index 58

more details

https://scholar.google.co.in/citations?user=Epk4eBkAAAAJ\&hl=en

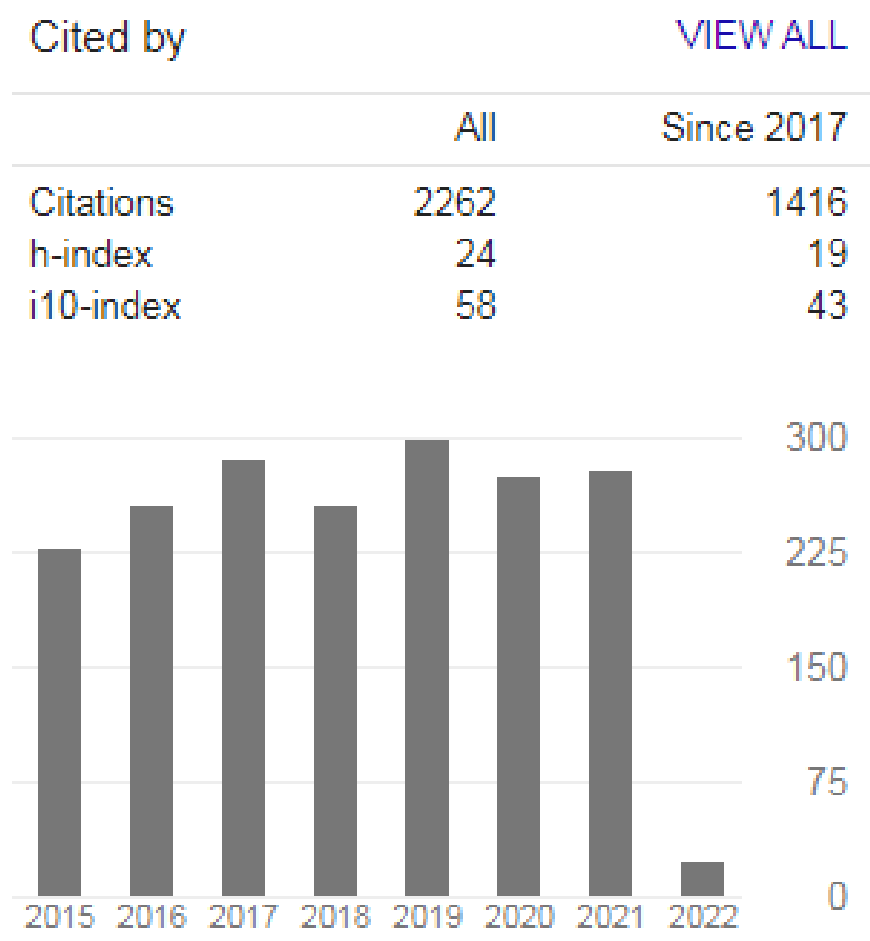

https://airccse.org/journal/ijmit/ijmit.html 


\title{
World Journal Clout Index (WJCI)
}

\author{
Impact Factor
}

2019- 0.211

2020- 0.040

\section{WJCI 2019}

https://wjci.cnki.net/UserIndex/JournalOverseaDetails?filename=ISSN09755926\&Year $=2019$

Ten 2019

\begin{tabular}{|c|c|c|c|c|c|}
\hline \multicolumn{2}{|c|}{ Jeurnal Fitle } & \multicolumn{4}{|c|}{ 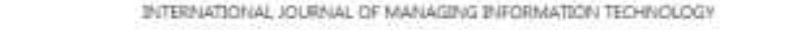 } \\
\hline \multicolumn{2}{|c|}{ Country/hogian } & \multicolumn{4}{|c|}{$10 \$ 2$} \\
\hline \multicolumn{2}{|r|}{ tasns } & \multicolumn{4}{|c|}{$0975-59260975-5585$} \\
\hline \multicolumn{2}{|c|}{ Publisher/sponsor } & \multicolumn{4}{|c|}{ A1R C C Publoking Corporation } \\
\hline \multicolumn{2}{|c|}{ Total Cites } & \multicolumn{4}{|c|}{30} \\
\hline \multicolumn{2}{|c|}{ Impaset foctor } & \multicolumn{4}{|c|}{0.231} \\
\hline \multirow{3}{*}{ Discipline Impat: } & Discipline & wjea & Dink & Quartile & Wja Peroentie \\
\hline & Information Procesting & Q 508 & $189 / 260$ & Q4 & 5.33 \\
\hline & Avenage Value & 0.280 & - & - & 5.48 \\
\hline
\end{tabular}




\section{WJCI 2020}

https://wjci.cnki.net/UserIndex/JournalOverseaDetails?filename=ISSN0975$5926 \&$ year $=2020$

Vow 2000

\begin{tabular}{|c|c|c|c|c|c|}
\hline \multicolumn{2}{|c|}{ Jeurnal titie } & \multicolumn{4}{|c|}{ 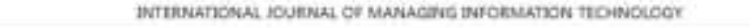 } \\
\hline \multicolumn{2}{|c|}{ Comarmryinogien } & \multicolumn{4}{|c|}{ India } \\
\hline \multicolumn{2}{|r|}{ ESS S } & \multicolumn{4}{|c|}{ D975-49260975-5596 } \\
\hline \multicolumn{2}{|c|}{ Putticren/Spomer } & \multicolumn{4}{|c|}{ ancc Futhithing Corpisation } \\
\hline \multicolumn{2}{|c|}{ Totat Cites } & \multicolumn{4}{|c|}{$z$} \\
\hline \multicolumn{2}{|c|}{ Impert Factor } & \multicolumn{4}{|c|}{20040} \\
\hline \multirow{3}{*}{ Discipline Impeca } & Dexiplien & wial & Tank & Quartie & wia Percentile \\
\hline & Informaties Processing & 0,021 & $173 / 276$ & Q4 & 270 \\
\hline & Average Velue & 0.051 & - & - & 170 \\
\hline
\end{tabular}

World Journal Clout Index $($ WJCI $)=$ WAJCI+WI $=0.011+0.080=0.091$ 